\title{
SMA-based Extraction of the Yeast Cell Wall Integrity Sensor Mid2 from Native Membranes of Saccharomyces cerevisiae - Electron Microscopy Study
}

\author{
Natalia Voskoboynikova ${ }^{1}$, Maria Karlova ${ }^{2}$, Rainer Kurre ${ }^{3}$, Juergen Heinisch ${ }^{4}$, Heinz-Juergen Steinhoff ${ }^{1}$, \\ Konstantin V. Shaitan ${ }^{1}$, Olga S. Sokolova ${ }^{1 *}$ \\ 1. School of Physics, University of Osnabrück, Osnabrück, Germany. \\ 2. Biology Faculty, Lomonosov Moscow State University, Moscow, Russia. \\ 3. Center of Cellular Nanoanalytics, Integrated Bioimaging Facility, University of Osnabrück, \\ Osnabrück, Germany. \\ 4. School of Biology, University of Osnabrück, Osnabrück, Germany. \\ * Corresponding author: sokolova@mail.bio.msu.ru
}

The single-span transmembrane Saccharomyces cerevisiae protein Mid2 is a member of the cell wall sensor family and is involved in programmed cell death [1]. Mid2 monitors cell integrity by signaling in response to the mating pheromone treatment, as well as during vegetative growth [2]. Mid2 is required for stress-induced nucleus-to-cytoplasm translocation of cyclin $\mathrm{C}$ in yeast [3]. In recent years, the progress of understanding the functioning of Mid 2 has been mostly limited to studies on living yeast cell systems $[4,5]$ or crude cell extract $[2,6,7]$. The use of green fluorescent protein (GFP)-tagged versions of Mid2 (Fig. 1) enabled following the distribution pattern of the sensor in the plasma membrane by quantitative live cell fluorescence [6]. However, no purification protocols for Mid2 have been described so far. In this work, we used amphipathic styrene-maleic acid (SMA) copolymers to solubilize GFPMid2 constructs into SMA lipid particles (SMALPs) from native yeast membranes, without the use of detergents. We characterized the obtained SMALP preparations by dynamic light scattering (DLS), fluorescence correlation spectroscopy (FCS) and single particle transmission electron microscopy (TEM).

We have developed a protocol for the direct extraction of the membrane protein Mid2-EGFP from the membrane fraction of $S$. cerevisiae cells by using SMA copolymers with a 3:1 molar styrene-to-maleic acid ratio. The DLS data (Fig. 2A) indicated the monodisperse nature of the SMALP preparations. By measuring the diffusion coefficient of the particles using FCS [8], we estimated the diameter of EGFPcontaining SMALPs as $8 \mathrm{~nm}$ (Fig. 2B). A SMALP sample (3 $\mu \mathrm{l})$ was placed onto a glow-discharged copper grid (Ted Pella, USA) and incubated at RT for $30 \mathrm{sec}$. Micrographs (Fig. 3A) were acquired using an analytical transmission electron microscope Jem-2100 (Jeol, Japan) equipped with a $2 \mathrm{~K} \times 2 \mathrm{~K}$ CCD camera Ultrascan 1000XP (Gatan, USA), using the SerialEM software in a low dose mode, with a magnification of $x 40000$ and a defocus of 0.5-1.9 $\mu \mathrm{m}$. The microscope was operated at $200 \mathrm{kV}$. The images were downloaded into the crYOLO [9] neuronal network and individual particles were automatically selected. The reference-free classification was implemented in RELION2.1 [10]. TEM allowed to analyze the morphology of SMALPs, compared to DLS and FCS methods. The obtained 2D classes represent the elongated particles, about $10 \mathrm{~nm}$ in length, that comprise several distinct domains (Fig. 3B).

We conclude that SMA copolymers could help to directly extract the transmembrane proteins from $S$. cerevisiae cells into the membrane mimetic nano-sized SMALPs. FCS analysis enabled for selective estimation of the size of the EGFP-Mid2-containing SMALPs; the size was comparable to that determined from the TEM data. The established protocol of detergent-free purification of membrane 
proteins from native yeast cells would enable in vitro structural and functional studies in a native-like membrane environment and in a single-particle manner [11].

References:

[1] R Rodicio and JJ Heinisch, Yeast 27 (2010), p. 531.

[2] M Rajavel et al., Mol Cell Biol 19 (1999), p. 3969.

[3] CY Jin et al., Oxid Med Cell Longev (2013),

[4] A Straede and JJ Heinisch, FEBS Lett 581 (2007), p. 4495.

[5] D Alsteens et al., Langmuir 28 (2012), p. 16738.

[6] C Kock et al., Cell Microbiol 18 (2016), p. 1251.

[7] T Ketela, R Green and H Bussey, J Bacteriol 181 (1999), p. 3330.

[8] Y Chen et al., Biophys J 82 (2002), p. 133.

[9] T Wagner et al., bioRxiv 356584 (2018).

[10] SH Scheres et al., J Mol Biol 348 (2005) p. 139.

[11] The authors acknowledge funding from the RFBR (\#18-504-12045 to K.V.S.) and DFG (\#STE640/15 to H.-J.S.). The JEOL2100 electron microscope is part of User facility center of MSU: "Electron microscopy in life sciences".

\begin{tabular}{|c|c|c|c|c|}
\hline signal & extracellular & TM & intracellular & GFP \\
\hline
\end{tabular}

Figure 1. Domain organization of Mid2.

\section{A}

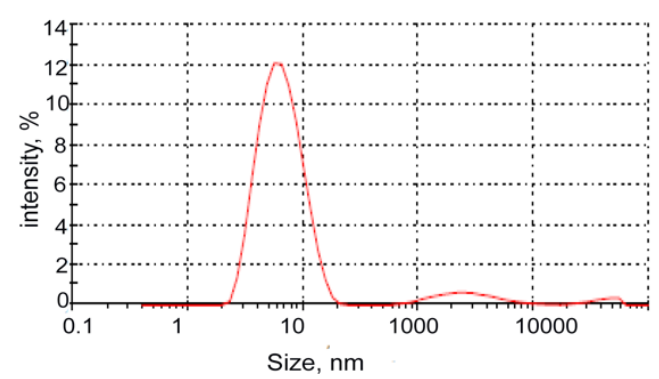

B

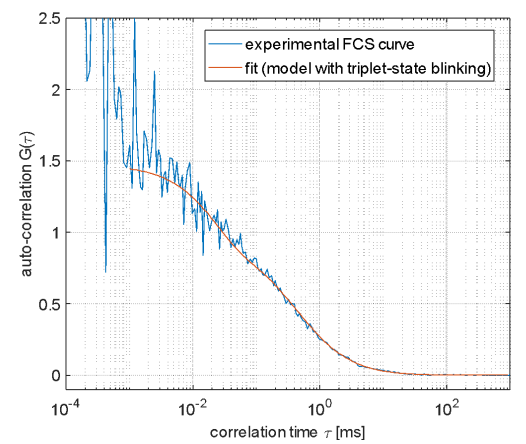

Figure 2. (A) Size distribution of the SMALPs, obtained by solubilization of the membrane fraction of S.cerevisiae cells. (B) FCS-curve (blue) of highly-diluted SMALP preparation in phosphate buffer saline. Curve was fitted (red) by a model considering triplet-state blinking. With the derived diffusion coefficient of $(59 \pm 3) \mu m^{2} / s$ a hydrodynamic radius of $r \approx 4 \mathrm{~nm}$ can be estimated by the StokesEinstein equation.
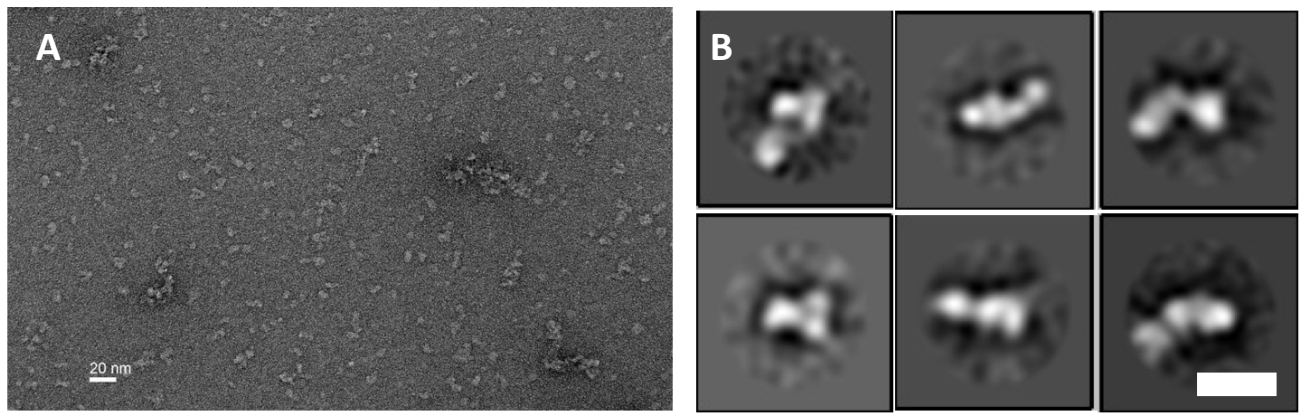

Figure 3. Negative stain TEM analysis of SMALP preparation. (A) raw image; (B) 2D images of selected SMALPs. Bar size - $10 \mathrm{~nm}$. 